\title{
Identification of a major rif transcript common to gametocytes and sporozoites of Plasmodium falciparum
}

\author{
Christian W Wang*1,2, Steven B Mwakalinga1,2, Colin J Sutherland³, Samana Schwank33, Sarah Sharp3, \\ Cornelus C Hermsen ${ }^{4}$, Robert W Sauerwein ${ }^{4}$, Thor G Theander ${ }^{1,2}$ and Thomas Lavstsen 1,2
}

\begin{abstract}
Background: The Plasmodium falciparum parasite is transmitted in its sexual gametocyte stage from man to mosquito and as asexual sporozoites from mosquito to man. Developing gametocytes sequester preferentially in the bone marrow, but mature stage gametocytes are released to the bloodstream. Sexual stage parasite surface proteins are of interest as candidate target antigens for transmission blocking vaccines.

Methods: In this study, the transcript profiles of rif and var genes, known to encode surface antigens in asexual blood stage parasites, were investigated at different stages of 3D7/NF54 gametocytogenesis and in sporozoites.

Results: Gametocytes exhibited a riftranscript profile unlinked to the rifand var transcript profile of the asexual progenitors. At stage V, mature gametocytes produced high levels of a single rifgene, PF13_0006, which also dominated the rif transcript profile of sporozoites. All var genes appeared to be silenced in sporozoites.

Conclusions: The most prominent variant surface antigen transcribed in both gametocytes and sporozoites of 3D7/ NF54 is a single variant of the RIFIN protein family. This discovery may lead to the identification of the parasites binding ligands responsible for the adhesion during sexual stages and potentially to novel vaccine candidates.
\end{abstract}

\section{Background}

Plasmodium falciparum is transmitted from infected humans to mosquitoes via sexual stages called gametocytes. Immature gametocytes arise from erythrocytic asexual stages and sequester preferentially in the bone marrow [1] for about 9-12 days before reaching maturity and emerging into peripheral blood [2]. Unlike other species of malaria parasites, five different stages of $P$. falciparum gametocyte development have been described in vitro by morphological criteria [2], known as stages I to $\mathrm{V}$. Only at stage $\mathrm{V}$ are gametocytes released to the bloodstream and become infectious to mosquitoes of the Anopheles sp. A mean circulation time of 6.4 days with a range of 1.3-22.2 days has been reported [3], but aggregation of the mature stage $\mathrm{V}$ gametocyte in the sub-dermal capillaries has been proposed [4,5]. In the mosquito

* Correspondence: cwang@sund.ku.dk

1 Department of International Health, Immunology, and Microbiology, University of Copenhagen, Copenhagen, Denmark

Full list of author information is available at the end of the article midgut, the male gametocyte transforms into eight motile microgametes and emerges from the erythrocyte within minutes (exflagellation), whereas the female emerges as a single round shaped macrogamete. Fertilization is required to initiate the mosquito stages of parasite development, resulting 10-18 days later in infectious sporozoites ready to invade a new human host.

It is thought that the sequestration during maturation of $P$. falciparum gametocytes allows avoidance of phagocytic clearance in the spleen, as immature sexual stages circulate in splenectomised hosts [6]. Several host receptors have been implicated in gametocyte adhesion, but at different stages of development. At stages I-IIA, CD36 seems to be the main receptor for adhesion [7-9], and at stages III-IV, ICAM-1, CD49c, CD166, and CD164 are candidate receptors [10]. Parasite ligands responsible for this sequestration are unknown, but identification of the relevant surface proteins is of great interest as they could be potential transmission blocking vaccine candidates $[11,12]$. 
Several multigene families encode proteins on the asexual parasite-infected erythrocytic surface such as PfEMP1, (Plasmodium falciparum erythocyte membrane protein 1), STEVOR (subtelomeric variable open reading frame) and RIFIN (repetitive interspersed family), and these are obvious candidates for studying gametocyte adhesion $[8,9,13]$. Hayward et al [8] found that the adhesion of early stage gametocytes to CD36 is mediated by PfEMP1, but not at later stages, where the knob structure is no longer present, and PfEMP1 seems to be confined to the parasite cytoplasm $[7,8]$.

PfEMP1 s (200-400 kDa) are encoded by approximately 60 var genes per genome. The clonal variant proteins are known to mediate cytoadhesion of the infected erythrocyte to host receptors [14] and are associated with development of immunity to asexual stages and protection from severe disease $[15,16]$. Based on sequence analysis, var genes were grouped in five different types $[17,18]$. A study of var transcript abundances in gametocytes showed a degree of var transcription programming favouring a subset of type $\mathrm{C}$ genes in gametocytes unlinked to the phenotype of asexual progenitors and unlinked to the transcription of stevor genes [13].

RIFIN proteins are encoded by the two-exon rif gene family of interspersed repetitive DNA, predominantly located in the sub-telomeric regions. Each parasite genome encodes around 200 genes encoding proteins of 30-40 kDa. RIFINs have conserved amino and carboxy termini, and one to two predicted transmembrane domains delineating a proposed extracellular variable region. No function has yet been ascribed to RIFINs. It has been proposed that RIFIN variants belonging to two disctinct subgroups designated A- and B-type RIFINs [19], show different subcellular localization patterns. Atype RIFINs were found to be transported to the surface of the infected erythrocyte and B-type RIFINs were retained inside the parasitophorous vacuole [20]. Furthermore, Petter et al [21] using a semiquantitative cDNA cloning approach provided evidence for stage-specific transcriptional regulation patterns during gametocytogenesis for the two rif-types. Expression of these multigene families has to date not been systematically investigated in sporozoites.

In this study, variant-specific real-time amplification was used to provide the first quantitative transcriptional analysis of each member of the rif gene family in both $P$. falciparum gametocytes and in sporozoites. A single rif gene was found to dominate transcription in both sporozoites as in gametocytes.

\section{Methods}

\section{Parasites}

Parasites of $P$. falciparum 3D7 and NF54 were cultured and induced to gametocytogenesis as previously described [13,22] at London School of Hygiene and Tropical Medicine, London, and at Radboud University Nijmegen Medical Centre, Nijmegen, respectively. RNA was taken from 3D7 gametocyte cultures on days 3, 5, and 10 and the gametocyte stages were determined as predominantly stage II, III, and V, respectively. RNA was taken from NF54 gametocyte culture at stage V. RNA was also taken from NF54 sporozoites isolated from the salivary glands of Anopheles sp. as previously described [23].

\section{RNA isolation and CDNA}

Parasites were harvested in Trizol reagent (Invitrogen) and total RNA was extracted according to the manufacturer's instructions (Invitrogen). Samples were treated with DNase I (Sigma) to digest any genomic DNA and tested in real-time PCR for contamination, using a primer set for the seryl-tRNA synthetase gene, p90. RNA was reverse transcribed from random hexamers, using Superscript II (Invitrogen), according to the manufacturer's instructions (Invitrogen). RNA from previous studies stored at $-80^{\circ} \mathrm{C}$ [13] was prepared for cDNA as described above. The RNA available was that taken from a 3D7 parasite line selected with IgG from semi-immune children, 3D7-Dodowa1, at the asexual ring stage and gametocyte stage III and from an unselected culture of clone 3D7 at the asexual ring stage, gametocyte stage II, III, and V.

\section{Primer design}

The rif primer pairs described previously [24], var primer pairs, and primers to the endogenous control genes seryltRNA synthetase and fructose-bisphosphate aldolase, p61, described previously [25] with modifications [26] were used.

\section{Quantitative PCR}

Quantitative PCR was executed on a Rotorgene RG-3000 thermal cycler (Corbett Research) applying QuantiTect SYBR Green PCR Master Mix (Qiagen) with primers at $20 \mu \mathrm{M}$, and internal control genes seryl-tRNA synthetase and fructose-bisphosphate aldolase, used for normalization as previously described [25]. Gene-specific standard curves were produced by determining the amplification efficiency relative to the single copy housekeeping gene, seryl-tRNA synthetase, based on quantitative measurements of 10-fold dilutions of genomic DNA and used to calculate the transcript copy number of each gene in tested cDNA.

\section{Results}

Rif gene transcript profiles in mature gametocytes and sporozoites is dominated by PF13_0006

Plasmodium falciparum parasites were induced to develop into sexual gametocyte stages and the RNA was isolated for transcriptional analysis. 3D7 gametocytes were induced in three separate experiments to isolate 
stage II, III and V, whereas NF54 stage V gametocytes were available for one experiment. In gametocytes, rif transcript abundances increased as the parasites matured and transcripts of PF13_0006 and PFI0025c progressively dominated, with PF13_0006 being the most dominant at gametocyte stage $\mathrm{V}$ (Figure 1). The transcript abundance of PF13_0006 was more than 100 times higher at gametocyte stage $\mathrm{V}$ than during the asexual stages (Figure 2). PF13_0006 also dominated the rif transcription in sporozoites (Figure 1).

\section{The gametocyte rif transcript profile is unlinked to the} phenotype of the asexual progenitors

Gametocyte stage parasites were induced from two different 3D7 lines. One unselected 3D7 and one 3D7 line selected with IgG from semi-immune children to select a variant surface antigen phenotype associated with severe malaria, 3D7-Dodowa1 [27]. The 3D7 and 3D7-Dodowa1 differed in their var transcript profiles at ring stage but showed similar var transcript profiles at gametocyte stages [13]. The rif transcript profiles of the two 3D7 lines also differed at ring stage parasites but appeared similar at the gametocyte stage III (Figure 1). RNA from later gametocyte stages of the selected line 3D7-Dodowa1 was not available.

\section{Var transcription in sporozoites}

The transcript abundance of var genes in sporozoites were investigated and showed that the transcript distribution was diverse and the levels were low (Figure 3) compared to those found in asexual ring stage- and gametocyte stage $\mathrm{V}$ parasites. Total transcript abundances of 0.4 (Figure 3), 55 and 3, respectively [13].

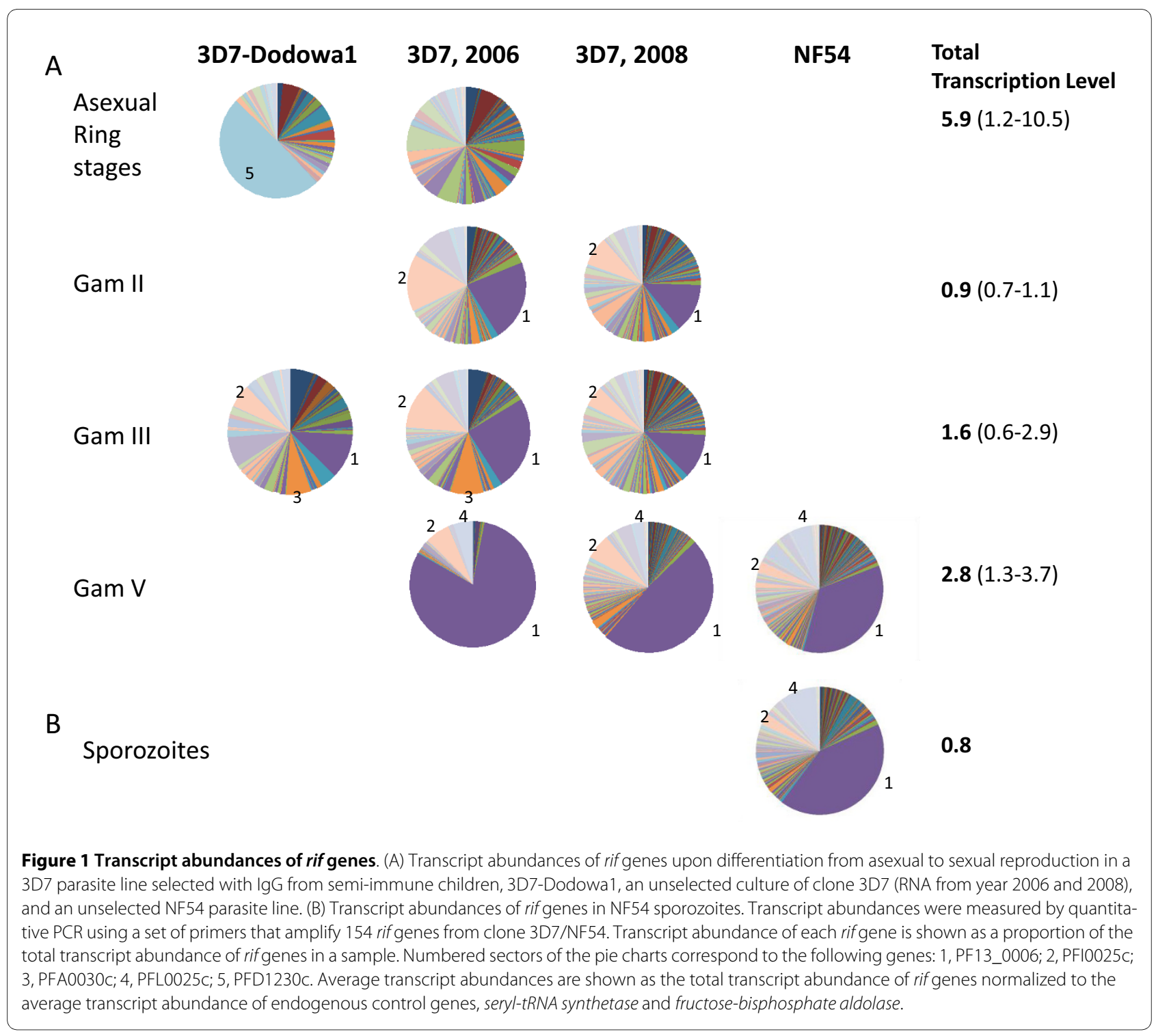




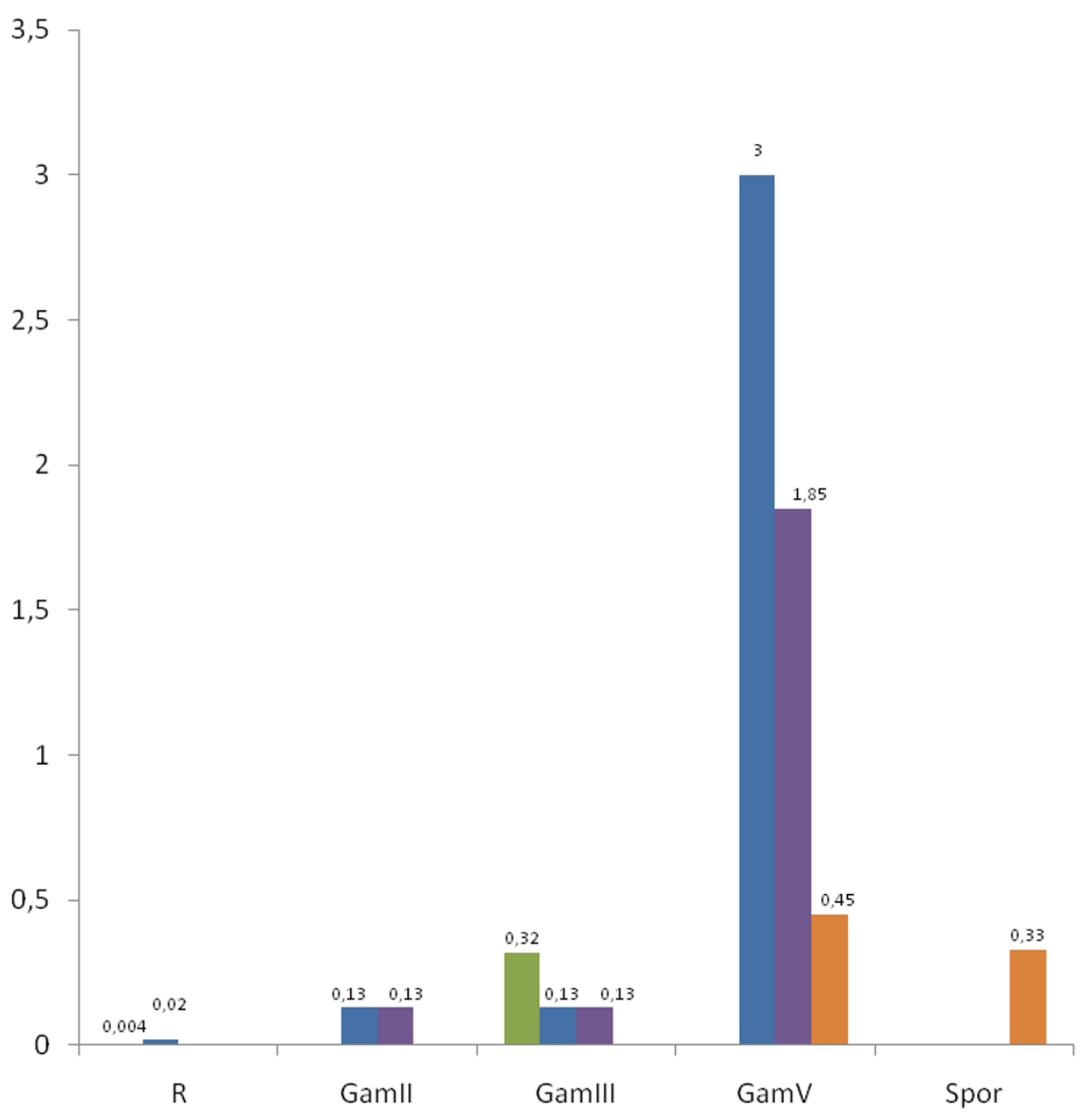

Figure 2 Transcript abundance of PF13_0006 during differentiation from asexual to sexual reproduction and in sporozoites. Transcript abundance of 1 corresponds to the mean level of two control genes, seryl-tRNA synthetase and fructose-bisphosphate aldolase. R, ring stage; Gamll, gametocyte stage II; GamIII, gametocyte stage III; GamIV, gametocyte stage IV; GamV, gametocyte stage V; Spor, Sporozoites. The colour codes identify the parasite culture from which RNA was purified.

\section{Discussion}

Antigens expressed on the surface of parasites and infected erythrocytes are attractive vaccine candidates. In this study, the transcription of variant gene families known to be expressed on the surface of infected erythrocytes was investigated. The data demonstrate a stage-specific transcription of rif genes in the developing gametocyte with up-regulation of a single rif gene: PF13_0006, in the mature stage V gametocytes. This result is supported by the study by Petter et al [21], who reported, using a plasmid clone counting approach, that the B1-type rif gene, PF13_0006 [19], but also PFI0025c, another B1-type rif gene dominated the rif gene transcript profile of developing stage II and III gametocytes. Here, we show that regardless of the rif and var transcript profile of the asexual progenitor, PF13_0006 dominates the transcript profile in gametocyte stages of both 3D7 and NF54 parasites lines. This implies that the encoded RIFIN has an important function in the developing gametocyte. However, expression studies of STEVOR in gametocytes have shown that the timing of protein expression and localisation may be uncoupled from that of transcription [28].

RIFINs could play a role in the sequestration of gametocytes, but the fact that the PF13_0006 transcript was maintained at high abundance in the non-adherent circulating stage $\mathrm{V}$ gametocytes makes it less likely that this protein is responsible for the sequestration. It is intriguing that PF13_0006 also dominated the rif transcript profile of sporozoites. It has previously been shown [24] that the transcription of PF13_0006 and PFI0025c during asexual blood stage development differs from that of 


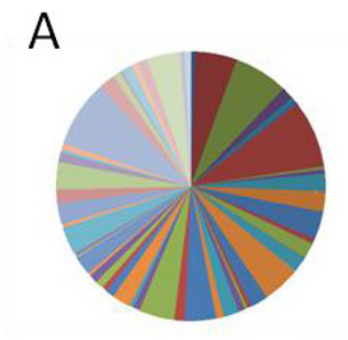

\section{B}

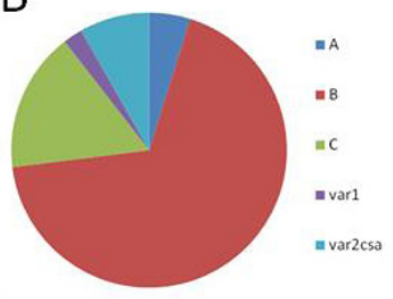

0.4

Figure 3 Transcript abundances of var genes. (A) Transcript abundances of each var gene shown as a proportion of the total transcript abundance of var genes in sporozoites of clone NF54. Transcript abundances were measured by quantitative PCR using a set of primers that amplify 59 var genes from clone 3D7/NF54. (B) Transcript abundances of each var gene shown as a proportion of the total transcript abundance of var genes and shown as proportion of var types according to the type described by Lavstsen et al [18]: A, B, C, var1, and var2csa. The total transcript abundance of var genes normalized to the average transcript abundance of endogenous control genes, seryl-tRNA synthetase and fructose-bisphosphate aldolase is shown. For comparison, asexual ring stage- and gametocyte stage $V$ parasites showed total transcript abundances of 55 and 3, respectively [13].

most other rif genes in that they dominated the transcript profile in the late schizonts and in the early rings. The profile was unlinked to the rif transcript profile during the mid ring and early trophozoite stages and the VSAphenotype of the parasite. This finding led to the suggestion that the proteins encoded by these two genes may be associated with the free merozoite stages. PF13_0006 may serve different functions depending on the developing pathway of the parasite; however, a common feature for extracellular gametes, sporozoites and merozoites is the need to "locate" another cell; sporozoites and merozoites in the bloodstream binding to hepatocytes and erythrocytes, respectively, and female and male gametes inside the mosquito midgut binding at fertilization. A role for RIFINs in these extracellular stages is at this point speculative but could include sensing, binding or both. However, localization of these RIFINs needs to be investigated, as surface expression on gametocytes, gametes, sporozoites, and merozoites has not as yet been established.

The transcription of rif genes is unlinked to var transcription in developing gametocytes and although PfEMP1 may have a role in sequestration of developing gametocytes to different host ligands, the function of RIFINs during gametocyte maturation remains to be confirmed.

\section{Conclusions}

In conclusion, this study has demonstrated that the rif transcript profile of gametocytes is unlinked to that of asexual progenitors. In particular a single rif gene,
PF13_0006, exhibits a consistently high transcript abundance in mature gametocyte stage $\mathrm{V}$ and in sporozoites. If homologues RIFINs of the B1-type are expressed on the surface of gametocytes, sporozoites, and merozoites of different origin this could lead to a possible vaccine candidate in all stages of the parasites life cycle.

\section{Competing interests}

The authors declare that they have no competing interests.

\section{Authors' contributions}

CWW carried out molecular biology studies, analysed data and wrote the paper. TL, SBM and TGT participated in the design, coordination and analysis of the study and helped to draft the manuscript. CJS, SS, and SS participated in the design and coordination of the gametocyte studies, helped analyse data and draft the manuscript. CCH and RWS participated in the design and coordination of the gametocyte and sporozoite studies, helped analyse data and draft the manuscript. All authors read and approved the final manuscript.

\section{Acknowledgements}

The study was supported by a Grant from the Foundation for the National Institutes of Health through the Grand Challenges in Global Health initiative. CWW was supported by the The Danish Medical Research Council (grant reference number 271-08-0540). The critical and highly professional support by Marga van de Vegte-Bolmer with gametocytes and Geert-Jan van Gemert for sporozoites are highly appreciated.

\section{Author Details}

1Department of International Health, Immunology, and Microbiology, University of Copenhagen, Copenhagen, Denmark, ${ }^{2}$ Department of Infectious Diseases, Copenhagen University Hospital (Rigshospitalet), Copenhagen, Denmark, ${ }^{3}$ Department of Infectious and Tropical Diseases, Immunology Unit, London School of Hygiene and Tropical Medicine, London, UK and ${ }^{4}$ Department of Medical Microbiology, Radboud University Nijmegen Medical Centre, Nijmegen, the Netherlands

Received: 26 April 2010 Accepted: 28 May 2010

Published: 28 May 2010

\section{References}

1. Smalley ME, Abdalla S, Brown J: The distribution of Plasmodium falciparum gametocytes in the internal organs and peripheral circulation. Trans R Soc Trop Med Hyg 1980, 75:103-105.

2. Hawking F, Wilson ME, Gammage K: Evidence for cyclic development and short-lived maturity in the gametocytes of Plasmodium falciparum. Trans R Soc Trop Med Hyg 1971, 65:549-559.

3. Eichner M, Diebner HH, Molineaux L, Collins WE, Jeffery GM, Dietz K Genesis, sequestration and survival of Plasmodium falciparum gametocytes: parameter estimates from fitting a model to malariatherapy data. Trans R Soc Trop Med Hyg 2001, 95:497-501.

4. Pichon G, Awono-Ambene HP, Robert V: High heterogeneity in the number of Plasmodium falciparum gametocytes in the bloodmeal of mosquitoes fed on the same host. Parasitol 2000, 121:115-120.

5. Gaillard FO, Boudin C, Chau NP, Robert V, Pichon G: Togetherness among Plasmodium falciparum gametocytes: interpretation through simulation and consequences for malaria transmission. Parasitol 2003, 127:427-435.

6. Bachmann A, Esser C, Petter M, Predehl S, von Kalckreuth V, Schmeidel S, Bruchhaus I, Tannich E: Absence of erythrocyte sequestration and lack of multicopy gene family expression in Plasmodium falciparum from a splenectomized malaria patient. PLoS One 2009, 4:e7459.

7. Day PD, Hayward RE, Smith D, Cuvenor JG: CD36-dependent adhesion and knob expression of the Transmission of Plasmodium falciparum is stage-specific. Mol Biochem Parasitol 1998, 93:167-177.

8. Hayward RE, Tiwari B, Piper KP, Baruch DI, Day KP: Virulence and Transmission success of the malaria parasite Plasmodium falciparum. Proc Natl Acad Sci USA 1999, 96:4563-4568.

9. Smith TG, Serghides L, Patel SN, Febbraio M, Silverstein RL, Kain KC: CD36mediated nonopsonic phagocytosis of erythrocytes infected with 
stage I and IIA gametocytes of Plasmodium falciparum. Infect Immun 2003, 71:393-400.

10. Rogers NJ, Hall BS, Obiero J, Targett GAT, Sutherland CJ: A model for sequestration of the transmission stages of Plasmodium falciparum: adhesion of gametocyte-infected erythrocytes to human bone marrow cells. Infect Immun 2000, 68:3455-33462.

11. Kaslow DC: Transmission-blocking vaccines: uses and current status of development. Int J Parasitol 1997, 27:183-189.

12. Sutherland CJ: Surface antigens of Plasmodium falciparum gametocytes - a new class of transmission-blocking vaccine targets? Mol Biochem Parasito/ 2009, 166:93-98.

13. Sharp S, Lavstsen T, Fivelman QL, Saeed M, McRobert L, Jensen AT, Baker DA, Theander TG, Sutherland CJ: Programmed transcription of the var gene family, but not of stevor, in Plasmodium falciparum gametocytes. Eukaryot Cell 2006, 5:1206-1214.

14. Kyes $S$, Horrocks $P$, Newbold C: Antigenic variation at the infected red cell surface in malaria. Annu Rev Microbiol 2001, 55:673-707.

15. Bull PC, Lowe BS, Kortok M, Molyneux CS, Newbold Cl, Marsh K: Parasite antigens on the infected red cell surface are targets for naturally acquired immunity to malaria. Nat Med 1998, 4:358-360.

16. Bull PC, Lowe BS, Kortok M, Marsh K: Antibody recognition of Plasmodium falciparum erythrocyte surface antigens in Kenya: evidence for rare and prevalent variants. Infect Immun 1999, 67:733-739.

17. Kraemer SM, Smith JD: Evidence for the importance of genetic structuring to the structural and functional specialization of the Plasmodium falciparum var gene family. Mol Microbiol 2003, 50:1527-1538.

18. Lavstsen T, Salanti A, Jensen ATR, Arnot DE, Theander TG: Sub-grouping of Plasmodium falciparum 3D7 var genes based on sequence analysis of coding and non-coding regions. Malar J 2003, 2:27.

19. Joannin N, Abhiman S, Sonnhammer EL, Wahlgren M: Sub-grouping and sub-functionalization of the RIFIN multi-copy protein family. $B M C$ Genomics 2008, 9:19.

20. Petter M, Haeggström M, Khattab A, Fernandez V, Klinkert MQ, Wahlgren M: Variant proteins of the Plasmodium falciparum RIFIN family show distinct subcellular localization and developmental expression patterns. Mol Biochem Parasitol 2007, 156:51-61.

21. Petter M, Bonow I, Klinkert MQ: Diverse expression patterns of subgroups of the rif multigene family during Plasmodium falciparum gametocytogenesis. PLoS One 2008, 3:e3779.

22. Ponnudurai T, Lensen AH, Meis JF, Meuwissen JH: Synchronization of Plasmodium falciparum gametocytes using an automated suspension culture system. Parasitol 1986, 93:263-274.

23. Lasonder E, Janse CJ, van Gemert GJ, Mair GR, Vermunt AM, Douradinha BG, van Noort V, Huynen MA, Luty AJ, Kroeze H, Khan SM, Sauerwein RW Waters AP, Mann M, Stunnenberg HG: Proteomic profiling of Plasmodium sporozoite maturation identifies new proteins essential for parasite development and infectivity. PLoS Pathog 2008, 4:e1000195.

24. Wang CW, Magistrado PA, Nielsen MA, Theander TG, Lavstsen T: Preferential transcription of conserved rif genes in two phenotypically distinct Plasmodium falciparum parasite lines. Int J Parasitol 2009, 39:655-664

25. Salanti A, Staalsoe T, Lavstsen T, Jensen ATR, Sowa MPK, Arnot DE, Hviid L, Theander TG: Selective upregulation of a single distinctly structured var gene in chondroitin sulphate A-adhering Plasmodium falciparum involved in pregnancy-associated malaria. Mol Microbiol 2003, 49:179-191.

26. Dahlbäck M, Lavstsen T, Salanti A, Hviid L, Arnot DE, Theander T, Nielsen MA: Changes in var gene mRNA levels during erythrocytic development in two phenotypically distinct Plasmodium falciparum parasites. Malar $J$ 2007, 6:78.

27. Jensen AT, Magistrado P, Sharp S, Joergensen L, Lavstsen T, Chiucchiuini A, Salanti A, Vestergaard LS, Lusingu JP, Hermsen R, Sauerwein R, Christensen J, Nielsen MA, Hviid L, Sutherland C, Staalsoe T, Theander TG: Plasmodium falciparum associated with severe childhood malaria preferentially expresses PfEMP1 encoded by group A var genes. J Exp Med 2004, 199:1179-1190

28. McRobert L, Preiser P, Sharp S, Jarra W, Kaviratne M, Taylor MC, Renia L, Sutherland CJ: Distinct trafficking and localization of STEVOR proteins in three stages of the Plasmodium falciparum life cycle. Infect Immun 2004, 72:6597-6602

doi: $10.1186 / 1475-2875-9-147$

Cite this article as: Wang et al., Identification of a major rif transcript common to gametocytes and sporozoites of Plasmodium falciparum Malaria Journal 2010, 9:147

\section{Submit your next manuscript to BioMed Central and take full advantage of:}

- Convenient online submission

- Thorough peer review

- No space constraints or color figure charges

- Immediate publication on acceptance

- Inclusion in PubMed, CAS, Scopus and Google Scholar

- Research which is freely available for redistribution

Submit your manuscript at www.biomedcentral.com/submit
C BioMed Central 